\title{
Constitutional mutation in CDKN2A is associated with long term survivorship in multiple myeloma: a case report
}

\author{
Vallari Shah ${ }^{*}$, Kevin D. Boyd ${ }^{2}$, Richard S. Houlston ${ }^{1,3}$ and Martin F. Kaiser ${ }^{1}$
}

\begin{abstract}
Background: Multiple Myeloma is a cancer of plasma cells associated with significantly reduced survival. Long term survivorship from myeloma is very rare and despite advances in its treatment the disease is generally considered incurable. We report a patient diagnosed with myeloma carrying a germline mutation of a tumour suppressor gene who has effectively been cured.

Case presentation: A 36-year-old woman was diagnosed with lgG lambda myeloma in 1985. She was treated with melphalan chemotherapy followed by high-dose melphalan and autologous stem cell rescue and since remained in complete remission despite not having received any additional therapy. After eliciting a prior history of multiple primary melanomas and breast cancer, she was tested for and shown to be a carrier for a germline mutation in CDKN2A.

Conclusions: This is the second case report of germline mutation of CDKN2A being associated with myeloma. CDKN2A is a stabiliser of p53. Long term survivorship after high dose DNA damaging chemotherapy with melphalan in this patient is compatible with an increased chemo-sensitivity due to impairment of the DNA repair pathway.
\end{abstract}

Keywords: Myeloma, Germline mutation, Survival, CDKN2A

\section{Background}

Multiple Myeloma (MM) is caused by the neoplastic proliferation of somatically mutated plasma cells and is associated with significant morbidity and mortality [1]. The use of alkylating agents such as melphalan to treat MM four decades ago led to the first appreciable improvement in patient outcome with survival rates of between 24 and 48 months after diagnosis [2]. The subsequent introduction of immunomodulatory agents, proteasome inhibitors and high-dose autologous stem cell transplantation, maintenance therapy, monoclonal antibodies and histone deacetylase inhibitors more recently has led to further improvements in patient outcome and median 5-year survival rates are typically now around 50\% [3]. Despite these advances in treatment autologous stem cell transplantation has still been shown to be beneficial in extending survival [4].

\footnotetext{
* Correspondence: Vallari.shah@icr.ac.uk

'Division of Molecular Pathology, The Institute of Cancer Research, London, UK

Full list of author information is available at the end of the article
}

There is however significant variation in outcome between patients with apparently same stage disease. Staging systems such as the international staging system (ISS) which uses serum albumin and $\beta 2$-microglobulin concentrations and the Revised-ISS (R-ISS) incorporating some adverse genetic markers and lactate dehydrogenase at diagnosis attempt to predict patients' outcome. These markers of adverse prognosis however cannot always accurately predict survival and there remain several factors that are currently unknown with regards to prognosis and response to treatment in myeloma. Of considerable interest is understanding why a very small number of patients have particularly long survivorship for what is essentially an incurable disease.

It is increasingly being recognised that, as well as the tumour profile, constitutional genotype also plays a role in determining patient outcome [5]. Here we report on an MM patient who has been in complete remission for over 30 years after only receiving first-line standard of care possibly being a consequence of also having hereditary Melanoma Syndrome. 


\section{Case presentation}

The patient, a 36-year-old woman, was diagnosed in 1985 with IgG lambda MM after presenting with tiredness and recurrent infections. She was found to be anaemic with a haemoglobin level of $73 \mathrm{~g} / \mathrm{l}$ and thrombocytopenic with a platelet count of $85 \times 10^{9} / \mathrm{l}$. Further testing revealed a markedly raised paraprotein of $62 \mathrm{~g} / \mathrm{l}$ with positive urinary Bence-Jones protein. There was evidence of immunosuppression with reduced levels of uninvolved IgA $(0.1 \mathrm{~g} / \mathrm{l})$ and IgM $(0.2 \mathrm{~g} / \mathrm{l})$ immunoglobulins. Her renal function was reduced as evidenced by a creatinine clearance of $57 \mathrm{ml} / \mathrm{min}$. A skeletal survey revealed multiple lytic lesions in both her humeri and femora. A bone marrow biopsy confirmed a diagnosis of MM.

Since the patient met the established criteria for symptomatic MM [6] with end organ involvement as demonstrated by her anaemia, bony lytic lesions and immunosuppression with recurrent infections, she was commenced on standard chemotherapy advocated at the time. This comprised three cycles of melphalan $10 \mathrm{mg}$ with prednisolone $60 \mathrm{mg}$ for 4 days orally. After 1 cycle of chemotherapy the patient's paraprotein had fallen to $26 \mathrm{~g} / \mathrm{l}$. She subsequently received two further cycles of melphalan and prednisolone which led to a further reduction in her paraprotein level to $7 \mathrm{~g} / \mathrm{l}$. This was followed by a high-dose melphalan $\left(140 \mathrm{mg} / \mathrm{m}^{2}\right)$ and autologous stem-cell transplant. Three months after her bone marrow transplant the patient's paraprotein was undetectable and has never been detected again (Fig. 1).

The patient had been annually reviewed since diagnosis and has remained in complete remission 30 years later with no further chemotherapy for her MM.

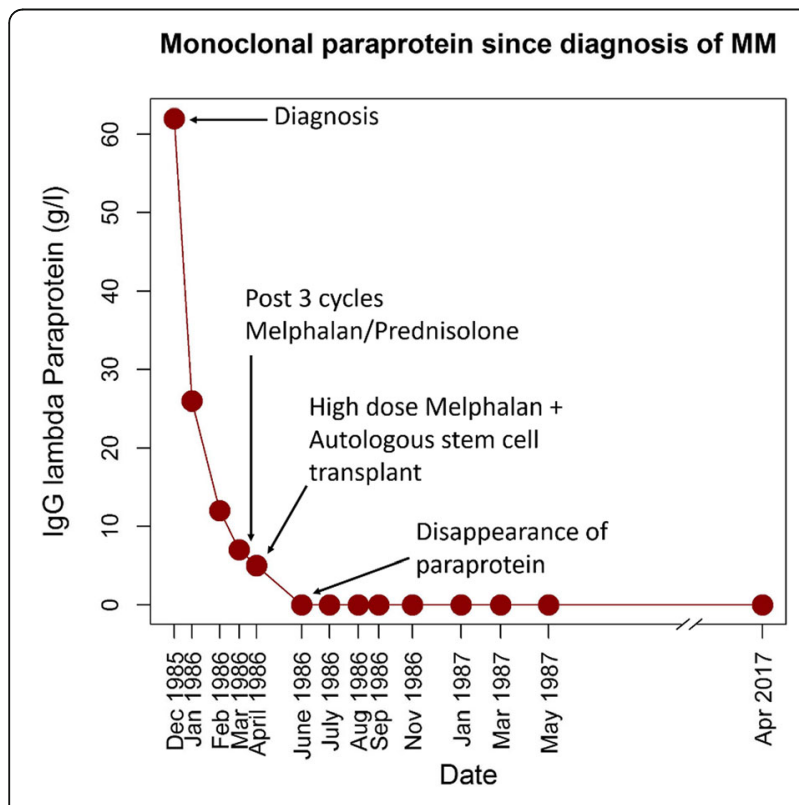

Fig. 1 Level of lgG lambda paraprotein ( $\mathrm{g} / \mathrm{l}$ ) from diagnosis of myeloma
Specifically, in April 2017, she continued to have a normal haematological profile with a haemoglobin of $135 \mathrm{~g} /$ l, white cell count of $9.1 \times 10^{9} / \mathrm{l}$, and a platelet count of $\left.191 \times 10^{9} / 1\right)$ ). She is no longer immunosuppressed with an IgA of $0.6 \mathrm{~g} / \mathrm{l}$ and IgM of $0.5 \mathrm{~g} / \mathrm{l}$. She also has no detectable paraprotein with a normal light chain ratio as assessed by serum protein electrophoresis and serum free light chain assay last assessed in April 2017. A repeat bone marrow biopsy 25 years ago showed that the patient was in complete remission. Imaging by whole body MRI in 2016 revealed no evidence of MM.

Five months prior to being diagnosed with MM she had been diagnosed with a superficial spreading malignant melanoma on her right leg, which was successfully treated by wide excision. She was subsequently diagnosed with three further primary melanomas at ages 53 (right buttock), 58 (right flank) and 62 (right forearm), all also successfully treated by surgical excision. While there was at that juncture no family history of melanomas or early onset pancreatic cancer, a diagnosis of hereditary melanoma syndrome which can be caused by germline mutations in the cyclin-dependent kinase Inhibitor 2A (CDKN2A) gene was considered in view of the history of multiple melanomas. Genetic testing of constitutional DNA extracted from EDTA venous blood was performed by genomic DNA PCR amplification using primers described previously of the 4 exons of CDKN2A (exons $1 \alpha, 1 \beta, 2$ and 3) [7]. PCR fragments were isolated by agarose gel electrophoresis and purified prior to Sanger sequencing using QIAquick Gel Extraction Kit (Qiagen, Paisley, UK). This revealed the patient was a heterozygous carrier of the pathogenic c.213C > A mutation in the CDKN2A gene. This mutation results in a missense substitution of the amino acid asparagine to lysine in the expressed INK4A protein at position $71(\mathrm{~N} 71 \mathrm{~K})$ and a leucine to methionine substitution in the expressed ARF protein (L86M) (Fig. 2). The patient's son has since been diagnosed with melanoma at the age of 34 years but he has yet to be genetically tested (Fig. 3). Otherwise the patient's family history is unremarkable and specifically there is no evidence for propensity to pancreatic cancers in family members.

Aside from MM and melanoma the patient has also been diagnosed with two other cancers. Firstly, in situ breast cancer at the age of 50 incidentally discovered during routine breast screening and which was treated with a mastectomy. Secondly, stage T2bN1M0 adenocarcinoma of the lung at the age 66 which was diagnosed following whole-body diffusion-weighted MRI investigation, performed as part of her MM follow-up investigation of hip pain. Her lung carcinoma has been treated by lobectomy, adjuvant chemotherapy with carboplatin and vinorelbine in addition to radiotherapy (Fig. 4). Mutation testing of the patient's lung cancer tissue by PCR 


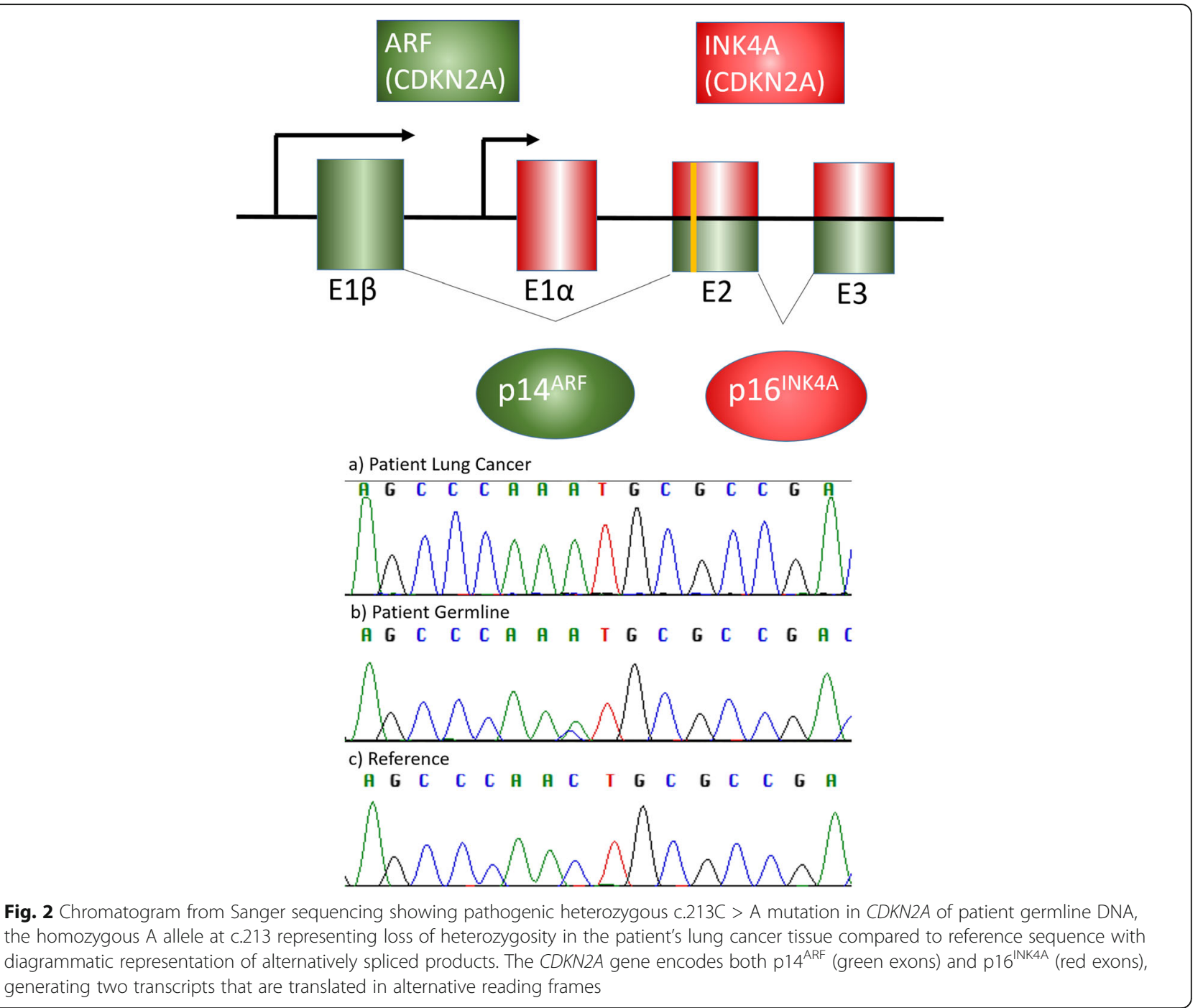

amplification and Sanger sequencing as described above revealed a loss of heterozygosity of the C.213C > A allele compared to the patient's germline DNA (Fig. 2). Paradoxically her MRI did not show any signs indicative of active MM.

\section{Discussion and conclusions}

The pathogenic nature of the specific c.213C > A mutation in CDKN2A noted in this patient is suggested by the fact that it has been described previously in several hereditary melanoma families [8-10] as well as a supraglottic squamous cell carcinoma [11]. In silico predictions with the algorithms used by Polyphen-2, SIFT and mutation taster all indicate that this is a pathogenic mutation. Additionally, functional assays of the protein INK4A with this mutation also suggest pathogenicity [12]. The loss of the wild type allele in the patient's lung cancer DNA as shown in Fig. 2 also suggests that this is a pathogenic mutation causing an increased susceptibility to tumours.
To our knowledge this is only the second case of a germline mutation in $C D K N 2 A$ being reported in association with MM. The previous report described a MM patient who had a strong family history of melanoma consistent with a diagnosis of hereditary Melanoma Syndrome caused by a pathogenic exon 1 CDKN2A mutation. Loss of the wild-type allele was detected in malignant plasma cells consistent with CDKN2A acting as a tumour suppressor in the context of MM in this case report [13].

Typically, germline mutation of $C D K N 2 A$ is associated with a restricted spectrum of cancers; primarily melanoma and pancreatic carcinoma. However, an increased risk of other cancers including childhood ones, lung, oropharyngeal and breast have been reported albeit at lower frequency [14]. Evidence for the association of the CDKN2A gene and its association with myeloma susceptibility has been shown in genome wide association studies which found a susceptibility locus for myeloma 


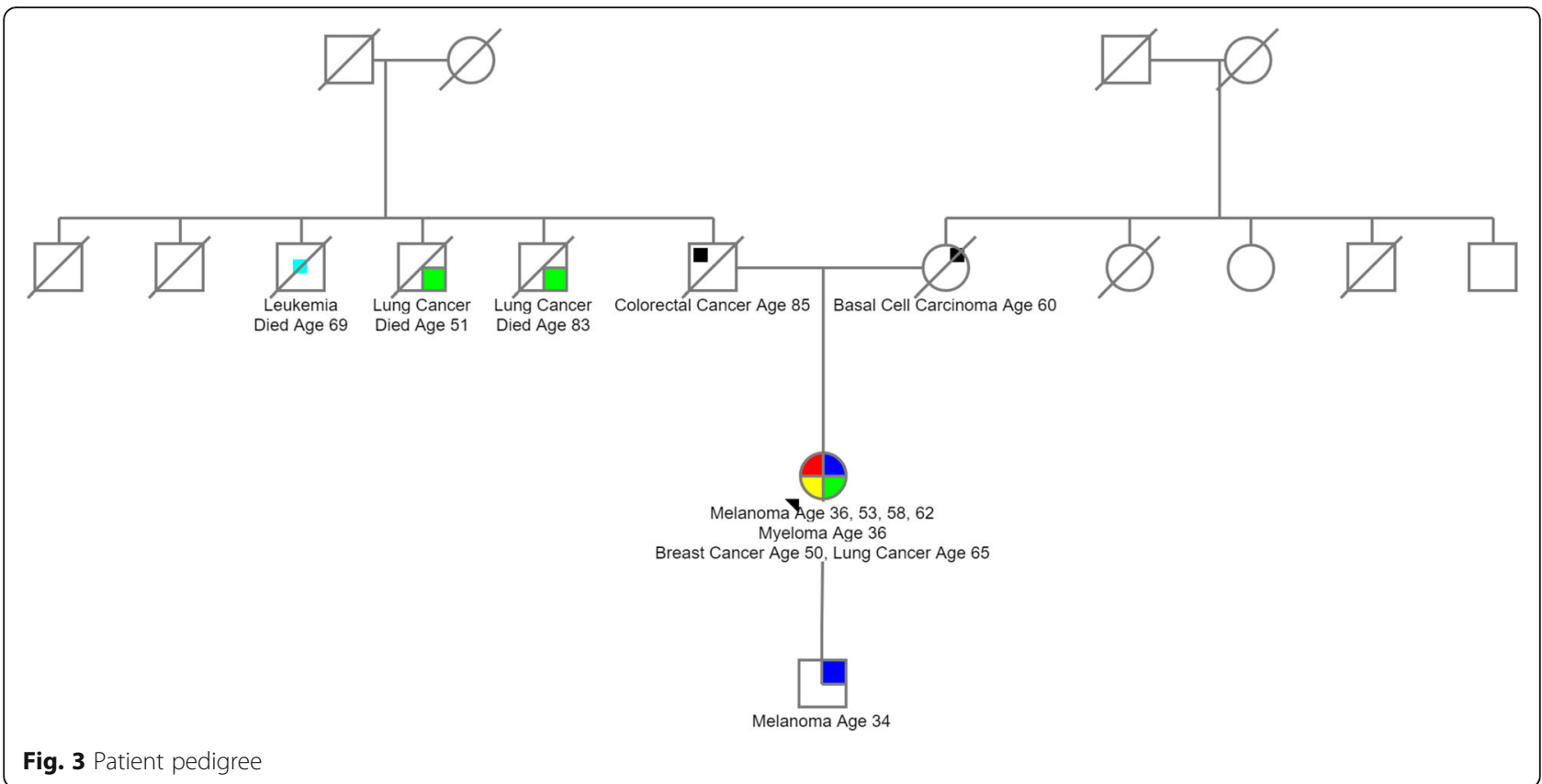

at chromosome 9p21.3 variant rs2811710 of CDKN2A [15]. A population based study in 1354 people with multiple myeloma also suggests a link between multiple myeloma, melanoma within first and second degree relatives [16]. This has been further confirmed in other studies [17-19].

Such data implies a wider impact of CDKN2A in tumour aetiology and although rare suggests the relationship with $\mathrm{MM}$ is not coincidental. It is perhaps not surprising that $C D K N 2 A$ impacts on the aetiology of a wide range of tumour types. One of the gene transcripts ARF functions as a stabiliser of p53 through interaction with E3 ubiquitin protein ligase MDM2, thereby enhancing p53-dependent transactivation and apoptosis. Mutations in ARF result in destabilisation of p53. Abnormalities of p53 are present in almost all cancers. This can be direct via deletion/mutation or hypermethylation of the p53 promoter, altering its stabilisation through alterations/ deletions of ARF or overexpression of MDM2 [20, 21] or via other mechanisms.

Alternate splice variant of CDKN2A, INK4A functions is a member of the cyclin dependent kinase inhibitors. It binds to CDK4 and CDK6 kinases and sequesters them from their regulatory cyclin D subunits. As a result, CDK4 cannot phosphorylate the retinoblastoma protein $(\mathrm{Rb})$ which is considered the gatekeeper of cell proliferation As a result of mutations in INK4A, there is resultant dysregulation of cell cycle control and tumour proliferation [22].

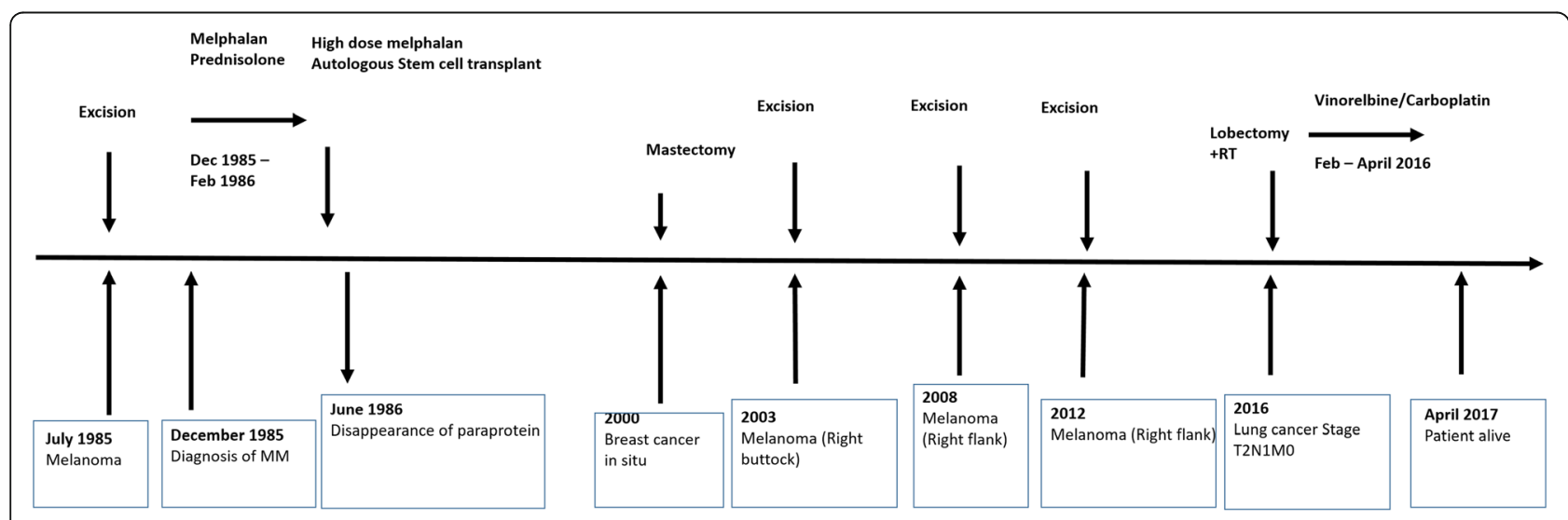

Fig. 4 Timeline of primary malignancies and therapy 
Further evidence of the role of $C D K N 2 A$ in tumour development comes from mice lacking ARF and/or INK4A which develop tumours early in life succumbing to lymphomas and fibrosarcomas [23]. Additionally, families with germline mutations of CDKN2A show increased rates of melanoma and pancreatic cancer but also have increased rates of other malignancies such as cancers of the breast, nervous system, GI tract, lymphoma and cervical cancers also suggesting that the increased susceptibility to cancer is not restricted to melanoma and pancreatic cancer alone [24, 25]. Furthermore, frequent somatic mutations and deletions of CDKN2A have been noted in several cancers including pancreatic adenocarcinoma, oesophageal and gastric carcinomas, leukemias and melanomas indicating its role in cancer pathogenesis $[26,27]$. Deletions as well as mutations of the $R b$ as well as TP53 are frequent in myeloma indicating a critical role of these genes in its pathogenesis [28]. It is therefore feasible that genes altering the function of these proteins will also increase the susceptibility to myeloma.

The case we report is striking in that after only melphalan therapy the patient has had a remission from $\mathrm{MM}$ of over 30 years and in essence is cured. Although speculative the observation is consistent with the patient's $M M$ being especially sensitive to alkylating chemotherapy. Melphalan causes DNA damage and subsequent cell death due to impairment in the DNA repair pathway. Studies have shown that mutations in $C D K N 2 A$ increase sensitivity to chemotherapy $[29,30]$. Moreover, MDM2 inhibitors increase sensitivity to conventional chemotherapy in different cancers. MDM2 targets p53 protein for proteosomal degradation and its function is inhibited by ARF. Mutations in ARF have been shown to destabilise p53 through this mechanism and the increased susceptibility to chemotherapy induced by MDM2 inhibitors may reflect why this patient also had a high sensitivity to conventional chemotherapy which causes DNA damage. Effectiveness of MDM2 inhibitors has also been demonstrated in haematological malignancies such as MM, AML and ALL. [31-33]. Of note in this regard is that AML with the translocation of $t(8 ; 21)$ resulting in the RUNX1-ETO fusion gene which directly inhibits ARF is one of the few subtypes of AML which can be cured by high dose chemotherapy alone [34].

In conclusion, outcome of high-dose chemotherapy in the patient we report has resulted in cure suggesting that such germline mutations may confer increased MM sensitivity to chemotherapy. This finding raises the possibility that long-term survivorship from $M M$ in other patients may be the consequence of carrier status for tumour suppressor genes with biological relevance to DNA damage.

\section{Abbreviations}

ALL: acute lymphoblastic leukemia; AML: acute myeloid leukemia; CDKN2A: cyclin-dependent kinase Inhibitor 2A; DNA: deoxyribonuelcic acid; EDTA: Ethylanediaminetatraacetic acid; MDM2: mouse double minute 2 homolog/E3 ubiquitin-protein ligase MDM2; MM: Multiple Myeloma; p53: tumour protein p53; RUNX1-ETO: Runt-related transcription factor 1Eight Twenty-One oncoprotein

\section{Acknowledgements}

We thank the patient for allowing us to write the case report. We also acknowledge support from the National Institute of Health Biomedical Research Centre at the Royal Marsden Hospital. We are grateful to Rebecca Brown from the Leeds Genetics Laboratory for her advice and guidance with regards to CDKN2A mutations. We would also like to thank Sidra Ellis, Fabio Mirabella and Karen Menezes for their help and advice in the lab.

\section{Funding}

None.

Availability of data and materials

Data is included within the article.

\section{Authors' contributions}

VS: design and draft of manuscript. KDB: design and draft of manuscript. RSH: concept, design and draft of manuscript. MFK: concept, design and draft of manuscript. All authors have read and approved the final version of the manuscript.

Ethics approval and consent to participate Not applicable.

\section{Consent for publication}

Written informed consent was obtained from the patient for publication of the case report.

\section{Competing interests}

The authors declare that they have no competing interests.

\section{Publisher's Note}

Springer Nature remains neutral with regard to jurisdictional claims in published maps and institutional affiliations.

\section{Author details}

${ }^{1}$ Division of Molecular Pathology, The Institute of Cancer Research, London, UK. ²Department of Haemato-Oncology, Royal Marsden Hospital, London, UK. ${ }^{3}$ Division of Genetics and Epidemiology, The Institute of Cancer Research, London, UK

Received: 27 June 2017 Accepted: 30 October 2017

Published online: 06 November 2017

\section{References}

1. Song X, Cong Z, Wilson K. Real-world treatment patterns, comorbidities, and disease-related complications in patients with multiple myeloma in the United States. Curr Med Res Opin. 2016:32(1):95-103.

2. Alexanian $\mathrm{R}$, et al. Treatment for multiple myeloma. Combination chemotherapy with different melphalan dose regimens. JAMA. 1969; 208(9):1680-5.

3. Rajkumar SV, Kumar S. Multiple myeloma: diagnosis and treatment. Mayo Clin Proc. 2016;91(1):101-19.

4. Rosinol $\mathrm{L}$, et al. Superiority of bortezomib, thalidomide, and dexamethasone (VTD) as induction pretransplantation therapy in multiple myeloma: a randomized phase 3 PETHEMA/GEM study. Blood. 2012:120(8):1589-96.

5. Johnson DC, et al. Genome-wide association study identifies variation at $6 q 25.1$ associated with survival in multiple myeloma. Nat Commun. 2016:7:10290

6. Rajkumar SV. Myeloma today: disease definitions and treatment advances. Am J Hematol. 2016:91(1):90-100.

7. Harland $M$, et al. A comparison of CDKN2A mutation detection within the melanoma genetics consortium (GenoMEL). Eur J Cancer. 2008;44(9):1269-74. 
8. Soufir $\mathrm{N}$, et al. Prevalence of p16 and CDK4 germline mutations in 48 melanoma-prone families in France. The French familial melanoma study group. Hum Mol Genet. 1998;7(2):209-16.

9. Bishop DT, et al. Geographical variation in the penetrance of CDKN2A mutations for melanoma. J Natl Cancer Inst. 2002;94(12):894-903.

10. Goldstein AM, et al. Features associated with germline CDKN2A mutations: a GenoMEL study of melanoma-prone families from three continents. J Med Genet. 2007;44(2):99-106.

11. Fischer M. Analysis of exon 2 of MTS1 in HPV-positive and HPV-negative tumors of the head and neck region. Eur Arch Otorhinolaryngol. 2007; 264(7):801-7.

12. McKenzie HA, et al. Predicting functional significance of cancer-associated p16(INK4a) mutations in CDKN2A. Hum Mutat. 2010;31(6):692-701.

13. Dilworth $D$, et al. Germline CDKN2A mutation implicated in predisposition to multiple myeloma. Blood. 2000;95(5):1869-71.

14. Mukherjee $B$, et al. Risk of non-melanoma cancers in first-degree relatives of CDKN2A mutation carriers. J Natl Cancer Inst. 2012;104(12):953-6.

15. Mitchell JS, et al. Genome-wide association study identifies multiple susceptibility loci for multiple myeloma. Nat Commun. 2016;7:12050.

16. Camp NJ, Werner TL, Cannon-Albright LA. Familial myeloma. N Engl J Med. 2008;359(16):1734-5. author reply 1735

17. Altieri $\mathrm{A}$, et al. Familial risks and temporal incidence trends of multiple myeloma. Eur J Cancer. 2006;42(11):1661-70.

18. Kristinsson SY, et al. Risk of solid tumors and myeloid hematological malignancies among first-degree relatives of patients with monoclonal gammopathy of undetermined significance. Haematologica. 2009;94(8):1179-81.

19. Lynch HT, et al. Familial multiple myeloma: a family study and review of the literature. J Natl Cancer Inst. 2001;93(19):1479-83.

20. Zhang Y, Xiong Y. Mutations in human ARF exon 2 disrupt its nucleolar localization and impair its ability to block nuclear export of MDM2 and p53. Mol Cell. 1999;3(5):579-91.

21. Zhang $Y$, Xiong $Y$, Yarbrough WG. ARF promotes MDM2 degradation and stabilizes p53: ARF-INK4a locus deletion impairs both the Rb and p53 tumor suppression pathways. Cell. 1998;92(6):725-34

22. Lin YC, et al. Human p16gamma, a novel transcriptional variant of p16(INK4A), coexpresses with p16(INK4A) in cancer cells and inhibits cellcycle progression. Oncogene. 2007;26(49):7017-27.

23. Kamijo T, et al. Tumor suppression at the mouse INK4a locus mediated by the alternative reading frame product p19ARF. Cell. 1997;91(5):649-59.

24. Borg A, et al. Novel germline p16 mutation in familial malignant melanoma in southern Sweden. Cancer Res. 1996:56(11):2497-500.

25. Hewitt $\mathrm{C}$, et al. Germline mutation of ARF in a melanoma kindred. Hum Mol Genet. 2002:11(11):1273-9.

26. Caldas $C$, et al. Frequent somatic mutations and homozygous deletions of the p16 (MTS1) gene in pancreatic adenocarcinoma. Nat Genet. 1994;8(1):27-32.

27. Liu Q, et al. MTS-1 (CDKN2) tumor suppressor gene deletions are a frequent event in esophagus squamous cancer and pancreatic adenocarcinoma cell lines. Oncogene. 1995;10(3):619-22.

28. Walker BA, et al. Mutational Spectrum, copy number changes, and outcome: results of a sequencing study of patients with newly diagnosed myeloma. J Clin Oncol. 2015;33(33):3911-20.

29. Simon M, et al. Role of p16 and p14ARF in radio- and chemosensitivity of malignant gliomas. Oncol Rep. 2006;16(1):127-32.

30. Iwadate $Y$, et al. Alteration of CDKN2/p16 in human astrocytic tumors is related with increased susceptibility to antimetabolite anticancer agents. Int J Oncol. 2000;17(3):501-5.

31. Andreeff $M$, et al. Results of the phase I trial of RG7112, a small-molecule MDM2 antagonist in leukemia. Clin Cancer Res. 2016;22(4):868-76.

32. Gu D, et al. Inhibition of the MDM2 E3 ligase induces apoptosis and autophagy in wild-type and mutant p53 models of multiple myeloma, and acts synergistically with ABT-737. PLoS One. 2014;9(9):e103015.

33. Reis $B$, et al. Acute myeloid leukemia patients' clinical response to idasanutlin (RG7388) is associated with pre-treatment MDM2 protein expression in leukemic blasts. Haematologica. 2016;101(5):e185-8.

34. Linggi $B$, et al. The $t(8 ; 21)$ fusion protein, AML1 ETO, specifically represses the transcription of the p14(ARF) tumor suppressor in acute myeloid leukemia. Nat Med. 2002;8(7):743-50.

\section{Submit your next manuscript to BioMed Central and we will help you at every step:}

- We accept pre-submission inquiries

- Our selector tool helps you to find the most relevant journal

- We provide round the clock customer support

- Convenient online submission

- Thorough peer review

- Inclusion in PubMed and all major indexing services

- Maximum visibility for your research

Submit your manuscript at www.biomedcentral.com/submit

) Biomed Central 